\title{
Propuesta de red de distribución para reciclaje de desechos eléctricos y electrónicos
}

\author{
José-Raúl Morales-Contreras, Santiago-Omar Caballero-Morales, \\ Patricia Cano-Olivos, Diana Sánchez-Partida \\ Universidad Popular Autónoma del Estado de Puebla A.C., \\ Departamento de Posgrado en Logística y Dirección de la Cadena de Suministro, \\ Puebla, México \\ joseraul.morales@upaep.edu.mx, santiagoomar.caballero@upaep.mx, \\ patricia.cano@upaep.mx, diana.sanchez@upaep.mx
}

\begin{abstract}
Resumen. La eliminación de desechos eléctricos y electrónicos, conocidos como tecno-basura, es uno de los retos más importantes para el medio ambiente. Esto dados los diferentes metales pesados que contienen estos desechos. Si bien existen centros especializados de reciclaje con la infraestructura para separar metales que pueden ser re-utilizados para reducir su impacto ambiental, su transporte hacia dichos centros es un problema que requiere planeación. El presente trabajo define una ruta logística para distribución de tecno-basura a 24 centros de reciclado en la Ciudad de Puebla. Esto con la finalidad de contribuir a la disminución de los niveles de tecno-basura en la ciudad. Para ello se emplearon herramientas heurísticas y programación en Octave. Los resultados respaldan la factibilidad de esta metodología en el contexto real para transporte de estos desechos.
\end{abstract}

Palabras clave: tecno-basura, red de distribución, heurísticas, optimización.

\section{Distribution Network Approach for Recycling of Electrical and Electronic Waste}

\begin{abstract}
The disposal of electrical and electronic waste, known as e-waste, is one of the most important challenges for the environment. This is due to the different heavy metals within this waste. Although there are specialized recycling centers with the infrastructure to separate metals that can be reused to reduce their environmental impact, the transportation of this waste to these centers is a problem that requires planning. This work defines a logistic route for the distribution of e-waste to 24 recycling centers in the City of Puebla. This is proposed to contribute to decrease the levels of e-waste in the city. To accomplish this, heuristic tools and programming with Octave were considered. The results support the feasibility of this methodology in the real context of transportation of e-waste.
\end{abstract}

Keywords: techno-trash, distribution network, heuristics, optimization. 


\section{Introducción}

Hoy en día el término tecno-basura (e-waste en inglés) es ampliamente utilizado y hace referencia a un conjunto de residuos que son considerados de alto riesgo para la salud por los compuestos tóxicas que despiden [17]. Entre estos se encuentran el mercurio, plomo, cadmio, níquel, selenio, arsénico, cromo y bromo, que si no son tratados adecuadamente se convierten en un riesgo para la salud además de contribuir a la emisión de gases de efecto invernadero [5,24].

Principalmente estos compuestos están presentes en computadoras, teléfonos celulares, televisores y electrodomésticos que han llegado al final de su vida útil [3]. La tecno-basura resultante ha tenido un crecimiento constante. Tan solo en México, una persona genera $2.5 \mathrm{~kg}$ por año y cerca del $50 \%$ se deposita en rellenos sanitarios o en tiraderos que no tienen un control de lo que se vierte en ellos [17].

Bajo este contexto se han realizado propuestas para facilitar la recolección y tratamiento de este tipo de residuos. En [2] se propuso un modelo de programación estocástica para el diseño de una red logística inversa bajo la cantidad de retorno, la relación de clasificación (calidad) y las incertidumbres de los costos de transporte asociada a posibles localizaciones de centros de recolección y centros de clasificado.

Por su parte, en [23] se analizó el impacto de la planeación de la capacidad sobre el ciclo de vida del producto para el funcionamiento de las dimensiones de sustentabilidad en la responsabilidad social de la logística inversa utilizando sistemas dinámicos.

En [16] se presentó el diseño de una red logística inversa para la recolección de residuos de equipo eléctrico y electrónico en la región española de Galicia. Para ello, se propuso un enfoque jerárquico en donde la primera fase consistió de la formulación de un problema de ubicación de instalación mediante programación lineal entera mixta. En la segunda fase se formuló el problema de enrutamiento de vehículos de recolección para dicha instalación con flota heterogénea y se resolvió mediante un algoritmo heurístico basado en ahorros. En la tercera fase se realizó un estudio de simulación de las rutas de recolección para evaluar el rendimiento general del sistema de recuperación.

En [15] se desarrolló un modelo lineal entero mixto para un diseño de red logística inversa basada en la huella de carbono. La función objetivo de este modelo resuelto mediante el software Lingo minimizó costos considerando la recolección, eliminación, transporte, apertura fija y costos de emisiones de $\mathrm{CO}_{2}$.

En [11] se abordó la problemática nacional de Brasil sobre los residuos sólidos debido a la falta de métodos y regulaciones acerca de los desechos electrónicos. Para ello se utilizaron metodologías como SODA (Strategic Options Development Analysis), PSM (Problem Structuring Methods), VFT (Value Focused Thinking Approach), SSM (Soft System Methodology) y SCA (Strategic Choice Approach) para análisis estratégicos y mejorar la toma de decisiones conjuntas en la cadena de suministro para los fines de procesamiento de desechos electrónicos.

En [22] se buscó minimizar el costo total asociado al transporte, el costo operativo, costo fijo para nuevas instalaciones, costo de disposición final y costo del relleno sanitario, así como optimizar los ingresos de la venta de materiales recuperados. Mediante su propuesta se determinó el número y la ubicación de los sitios de almacenamiento y desmontaje necesarios con un incremento en la tasa de recuperación del $20 \%$. Por otra parte, en [1] se determinaron los centros de recolección necesarios 
para recolectar productos usados cuyo valor puede ser recuperado mediante procesos especiales. Esto puede motivar a las empresas a participar en las operaciones de recolección [14].

Otro trabajo relevante es el presentado en [18] en donde se identificaron y analizaron criterios para seleccionar socios en cadenas de suministro inversas para los fines de recolección y tratamiento de desechos. Para ellos se utilizó una metodología integrada por herramientas como AHP (Analytic Hierarchy Process) y VIKOR (del serbio VIseKriterijumska Optimizacija I Kompromisno Resenje o Multicriteria Optimization and Compromise Solution).

En [21] se propuso un sistema de apoyo al proceso de decisión que permitiera identificar el tipo de sistema o red más adecuada para la recuperación de productos fuera de uso. De esta manera se determinó que productos cuyo valor es más susceptible al paso del tiempo suelen requerir una configuración de red que permita recuperar el producto en un plazo de tiempo corto (responsiva). En tanto, si el valor del producto no es tan sensible al tiempo se puede tener un plazo de tiempo mayor para su procesamiento. También se consideraron los procesos de recuperación económica más apropiados a cada producto como reutilización, re-fabricación, y reciclaje.

En cuanto a la aplicación de modelos de ruteo son escasos los trabajos reportados que implementen, por ejemplo, el modelo del Problema de Ruteo Vehicular Capacitado (CVRP) específicamente para el diseño de redes de logística inversa. Existen recopilaciones como en [4] que presentan diferentes métodos matemáticos para la solución óptima de redes de distribución haciendo énfasis en las emisiones en las rutas óptimas.

En una dirección similar, existen trabajos que consideran el CVRP considerando flotas de vehículos híbridos (eléctricos) como soporte a una red logística verde [25]. El CVRP, al ser considerado como un problema de complejidad computacional NP-duro, usualmente requiere de métodos heurísticos para su resolución con instancias de mediana y alta escala. En el caso de [25] se utilizó un algoritmo de recocido simulado.

Por último, en [13] se integró el costo económico y la reducción del costo de emisión para un problema de enrutamiento de vehículos verdes con múltiples depósitos capacitados. Para esto se ocupó un modelo de programación lineal entera para un conjunto de instancias a pequeña escala utilizando la herramienta Lingo. Cabe mencionar que también se desarrolló una meta-heurística basada en la optimización de colonias de hormigas (ACO, por sus siglas en inglés) para resolver instancias de problemas tanto a pequeña como a gran escala en un tiempo razonable.

El presente trabajo aporta en el contexto de diseño de una red de transporte mediante el CVRP para la recolección de desechos electrónicos y su distribución a centros de procesamiento para la recuperación de su valor y correcta eliminación. Esto dado que, al igual que en lugares como España, Brasil, Turquía, en México hay mucha generación de este tipo de desecho y una tasa muy baja de eliminación responsable mediante el reciclado (5.1\% al año 2013). Actualmente, México se considera el segundo país de América Latina con más desechos eléctricos y electrónicos [19,20].

En el caso particular del Estado de Puebla, se recolectan en promedio 120 unidades de residuos electrónicos y eléctricos diario, siendo el cuarto estado con mayor recolección de este tipo de residuos a nivel nacional [12]. Sin embargo, estudios han comprobado que este tipo de desechos denominados tecno-basura ha superado la capacidad de reciclaje de los servicios sanitarios de la entidad [10]. 
Por lo tanto, se plantea que el presente trabajo brinde soporte al cuidado del medio ambiente en la entidad al proveer de una metodología para el diseño de red de recolección y distribución para el procesamiento correcto de tecno-basura. Esto con el fin de evitar que los desechos terminen en barrancas, rellenos sanitarios o tiraderos clandestinos, y que los compuestos dañinos que emiten no tengan un contacto directo con los recursos naturales.

\section{Contexto del problema}

En la zona metropolitana de Puebla existen 3 empresas autorizadas para el reciclado de residuos mismas que no son suficientes para el tratamiento de hasta 187 mil 677 toneladas que se generan de forma mensual [6]. Por lo que, la Secretaría de Desarrollo Rural, Sustentabilidad y Ordenamiento Territorial (SDRSOT) ha tenido que aprobar las autorizaciones de otros centros de recolección de empresas o centros de reciclaje privado para el manejo especial de residuos, es decir, para la separación y tratamiento de basura reciclable [6].

Sin embargo, a pesar de haber aumentado los centros de reciclado, estos se enfocan en el tratamiento de los residuos que les lleva la población, por lo que no existe un sistema de distribución que envíe los desechos de los Servicios Sanitarios de Puebla a los centros de reciclado para disminuir la acumulación de residuos eléctricos y electrónicos del establecimiento. Esto, a pesar de existir legislaciones que obligan a las entidades federativas a hacerse cargo de la recolección, acopio, almacenamiento, transporte, tratamiento y disposición final de la basura en general [8].

Bajo este contexto, se sugiere el diseño de una red de logística inversa que considere el transporte de residuos electrónicos y electrodomésticos desde la ubicación de los Servicios Sanitarios de Puebla que es en donde se concentra la mayor cantidad de residuos de la ciudad hasta los principales centros de reciclado de tecno-basura por medio de vehículos sujetos a cierta capacidad. Esto para el uso más eficiente de los recursos estatales o de empresas privadas que deseen participar en este proceso.

\section{Metodología}

\subsection{Obtención de datos}

Datos concernientes a las ubicaciones de los centros de reciclaje disponibles se basó en la información principalmente de la zona metropolitana de Puebla y zonas cercanas. Para el caso de estudio de la zona metropolitana del Estado de Puebla, la recolección de datos fue llevada a cabo mediante una minuciosa investigación en diferentes bancos de datos disponibles para el acceso público, a través de dos fuentes oficiales que son el INEGI y la Secretaría de Desarrollo Rural, Sustentabilidad y Ordenamiento Territorial (SDRSOT), de donde se obtuvo la información sobre las empresas que cuentan con permiso para el procesamiento de los residuos de aparatos eléctricos y electrónicos (RAEE). De esta manera la figura 1 presenta los centros identificados. 


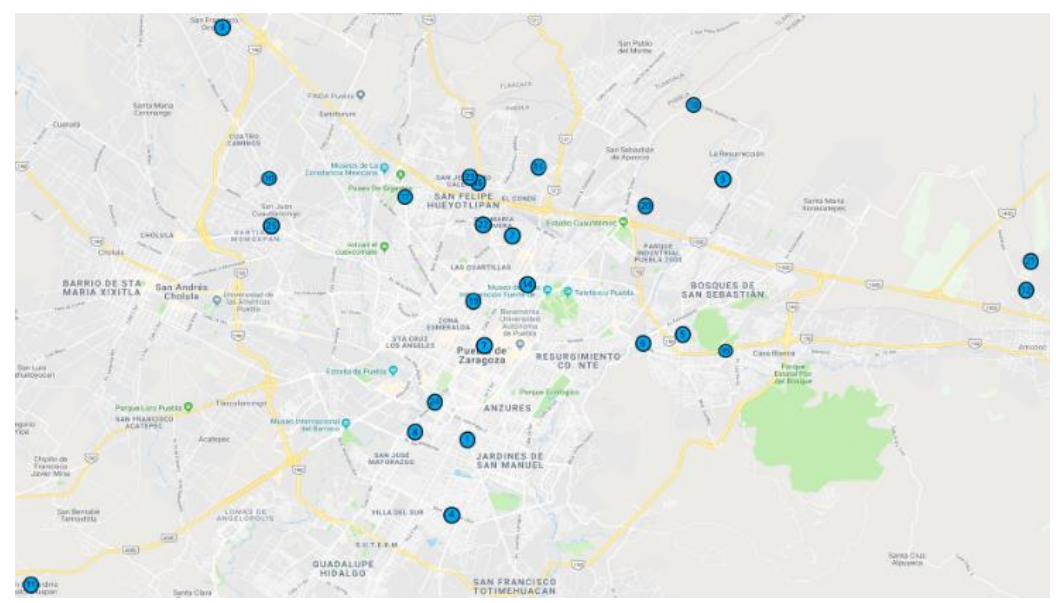

Fig. 1. Localización de 24 centros de reciclado y un centro de distribución en la zona metropolitana de Puebla.

A partir de esta información se estimó la llegada de desechos máxima a recibir por cada centro. Dado que el presente trabajo está enfocado en los desechos eléctricos y electrónicos, se consideraron las estadísticas más recientes con respecto a este rubro.

Al año 2011 se reportó que en México se generaban aproximadamente 300,000 toneladas de tecno-basura. Siendo el valle de México aquél con la mayor generación anual con 94,000 toneladas en tanto que las demás entidades generaban entre 36,000 y 10,000 toneladas al año [17]. Con una tasa estimada de crecimiento de $6 \%$ anual esto daría al año 2019 un aproximado de 451,090 toneladas. En el caso de Puebla se considera una cantidad aproximada de 12,000 toneladas anuales.

En cuanto a la capacidad de los vehículos para transporte se ha reportado un aproximado de entre 8 y 10 toneladas [7].

\subsection{Procesamiento de datos}

Para poder resolver el problema de ruteo es necesario hacer un procesamiento inicial de los datos. En primer lugar, se determinó la distancia relativa entre cada centro de reciclado y el centro de distribución. Para ello, se hizo uso de la distancia Euclidiana y de programación en Octave. La figura 2 presenta el código realizado para la obtención de este recurso en tanto la figura 3 presenta la matriz simétrica de distancias obtenida.

En segundo lugar, se estimó la llegada promedio diaria de toneladas a reciclar en cada centro de reciclado. Esto para hacer una eficiente la distribución de los desechos a lo largo del mes.

Previamente se estimó que la entidad de Puebla genera un aproximado de 12,000 toneladas anuales de tecno-basura. Esto es equivalente a 1000 toneladas al mes lo cual daría un estimado de 1000/30 días = 33.3 toneladas diarias, o 33.3 toneladas $/ 24$ centros $=1.38$ toneladas diarias a recibir por cada uno de los centros de reciclado. Para la capacidad de vehículos se estiman 8 toneladas de carga [7] 


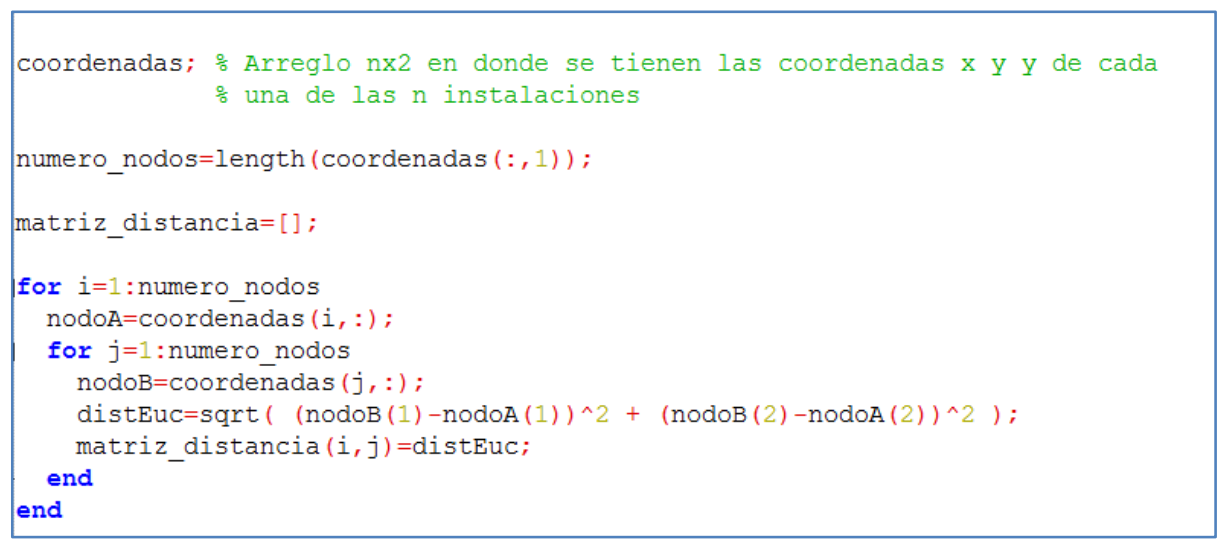

Fig. 2. Código Octave para estimación de matriz de distancias Euclidianas entre cada una de las 25 localizaciones de la red.

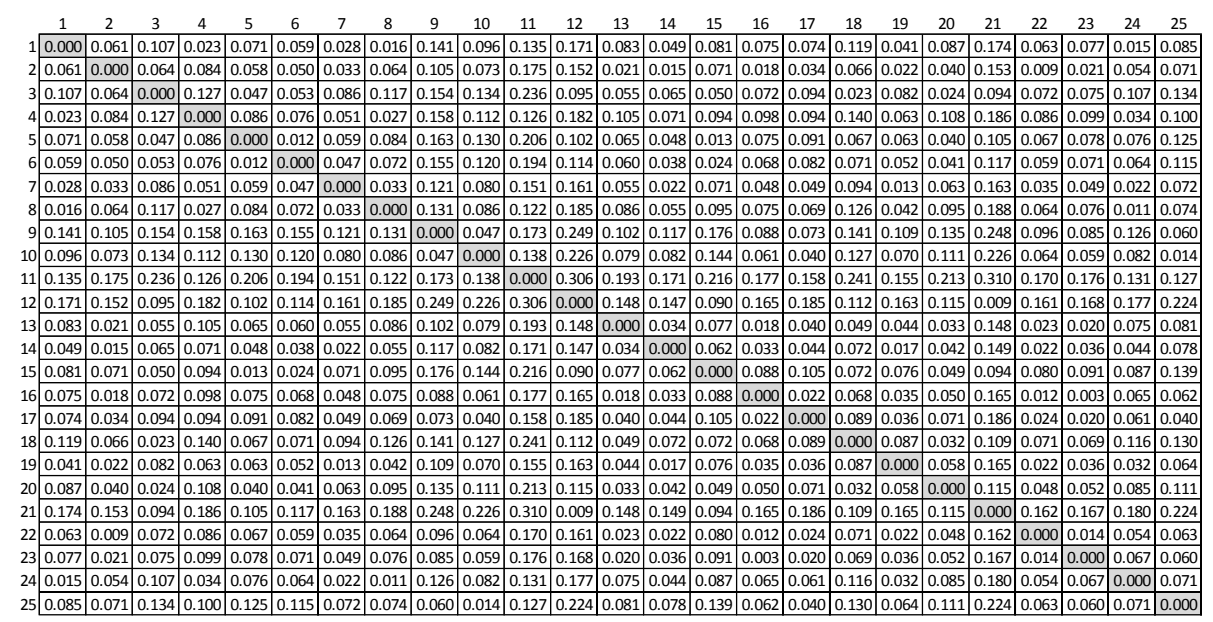

Fig. 3. Matriz de distancias entre cada instalación. En la localización 1 se identifica el centro de distribución en tanto que en las localizaciones 2-25 se identifican los 24 centros de reciclado.

\subsection{Método de solución}

Como se revisó en la Sección 1, muchos de los trabajos en el aspecto de redes para reciclado y procesamiento de desechos consideran el problema de ruteo vehicular o CVRP. Este problema se formula matemáticamente de la siguiente manera [9]:

$$
\min \sum_{i \in V} \sum_{j \in V} C_{i j} X_{i j} .
$$

Sujeto a:

$$
\sum_{i \in V} X_{i j}=1, \forall j \in V \backslash\{0\},
$$




$$
\begin{gathered}
\sum_{J \in V} X_{i j}=1, \forall i \in V \backslash\{0\}, \\
\sum_{i \in V} X_{i 0}=K, \\
\sum_{j \in V} X_{0 j}=K, \\
\sum_{i \notin S} \sum_{j \in S} X_{i j} \geq r(S) \forall S \subseteq V \backslash\{0\}, S \neq \varnothing, \\
X_{i j} \in\{0,1\}, \forall i, j \in V .
\end{gathered}
$$

En donde $C_{i j}$ es la distancia entre cada localización $i$ y $j, X_{i j}$ es la variable de decisión binaria que es igual a " 1 " si el camino entre $i$ y $j$ es parte de la solución y es igual a " 0 " en caso contrario, $K$ es el número de rutas / vehículos a considerar para dar servicio a todas las instalaciones, $V$ es el conjunto de nodos en la red en donde "0" es el centro de distribución, y $S$ es el subconjunto de nodos de $V$ que pertenece a las ubicaciones a visitar (centros de reciclado). De esta manera, (1) establece la función objetivo que minimiza los tiempos de ruteo entre cada una de las instalaciones en la red, (2) y (3) son restricciones que establecen que cada centro o nodo cliente (p.e., centros de reciclado) es visitado sólo una vez, las restricciones (4) y (5) establecen que los vehículos que salen del centro de distribución son los mismos que regresan al final de sus rutas de distribución. Finalmente, (6) es la restricción asegura que haya conectividad entre cada ubicación y (7) la establece la naturaleza de la variable de decisión.

En base a esta formulación, el resultado consistirá de rutas que en su conjunto darán servicio a cada nodo en la red, cubriendo sus requerimientos sin exceder la capacidad de los vehículos al mismo tiempo que se minimizara el tiempo o distancia de traslado entre los mismos. La figura 4 ilustra esta solución.

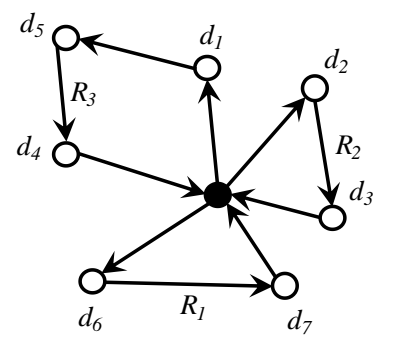

Fig. 4. Modelo de ruteo CVRP.

Para obtener la solución al CVRP se realizó la programación de una adaptación del meta-heurístico presentado en [9] mediante el software Octave. Nuestra adaptación se presenta en la figura 5 .

Este meta-heurístico fue considerado dado que puede implementarse de una manera rápida mediante software libre como lo es Octave. Esto en contraste con el uso de software que requiere algún tipo de licencia. 
A diferencia del meta-heurístico presentado en [9], nuestra adaptación incluye mejora en las dos etapas del mismo, esto es, en la creación de una solución inicial y en la obtención de la solución final.

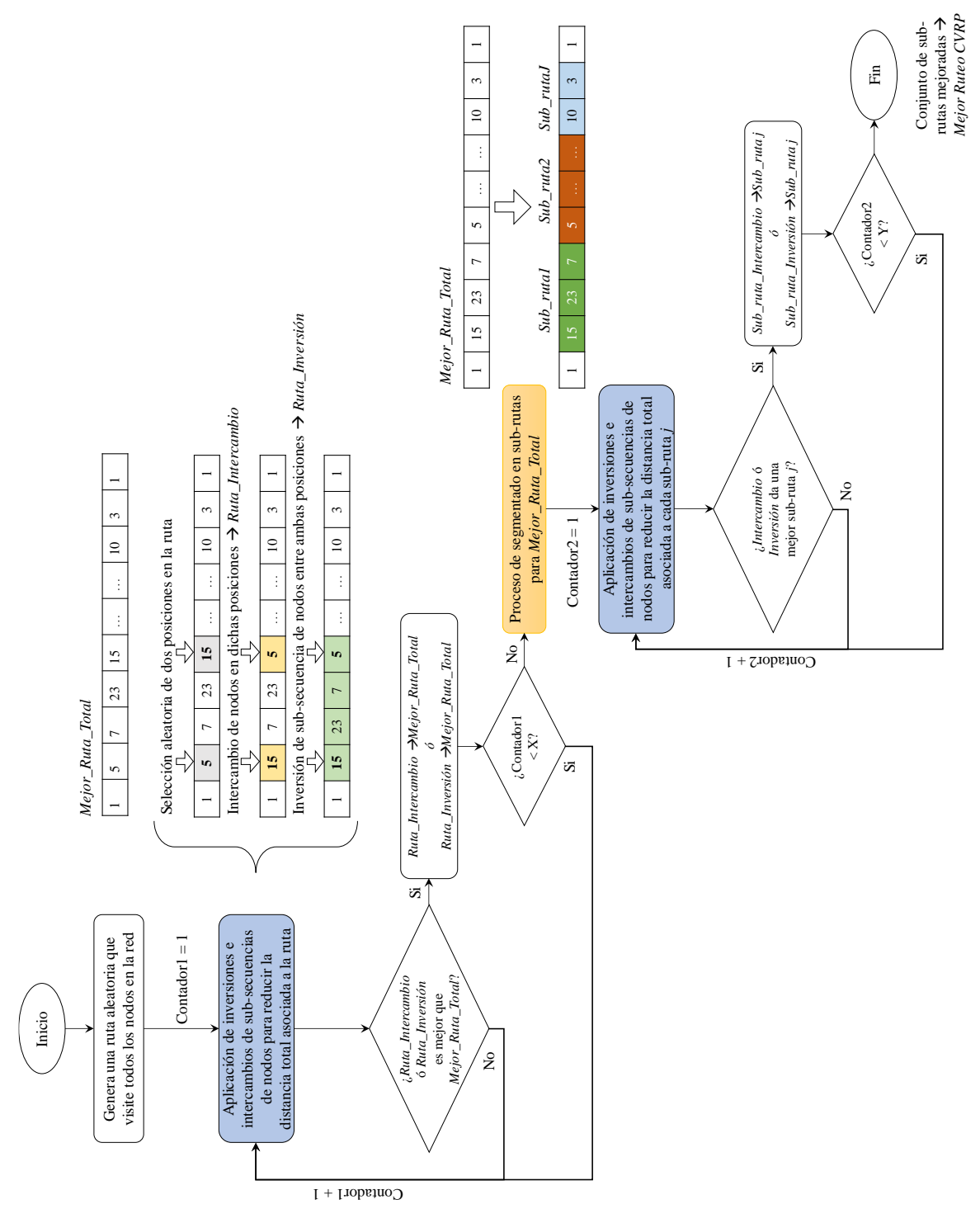

Fig. 5. Meta-heurístico implementado para dar solución al CVRP.

De manera general este meta-heurístico inicialmente crea una sola ruta para visitar a todos los centros de reciclado. Esto se hace sin considerar los requerimientos de los centros y las capacidades de los vehículos. Esta ruta se mejora mediante operaciones heurísticas de inversión e intercambio de nodos/subsecuencias de nodos, que son 
algoritmos heurísticos para el mejoramiento de una solución. En medida que se aplican estos procesos se obtiene una ruta cuya distancia total sea mínima.

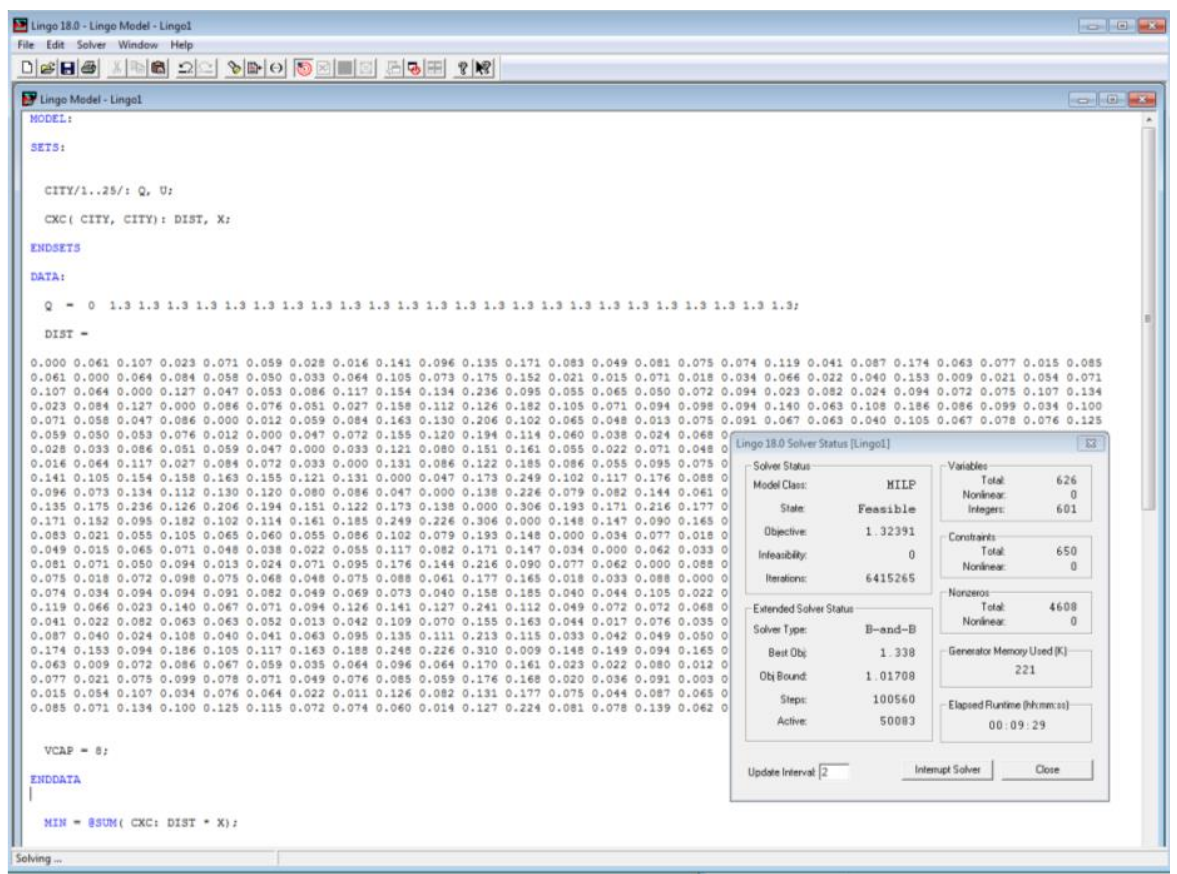

Fig. 6. Implementación en Lingo para evaluar el desempeño del meta-heurístico para el CVRP.

La mejor ruta obtenida después de $\mathrm{X}=100,000$ iteraciones de los procesos de inversión/intercambio se utilizó para la siguiente etapa que consiste en el segmentado en sub-rutas. En esta etapa se consideran las restricciones de demanda y capacidad de los centros y vehículos respectivamente. Al igual que en la etapa inicial de creación, las sub-rutas segmentadas se mejoran mediante intercambio e inversión de nodos. Este proceso se ejecutó durante $\mathrm{Y}=10,000$ iteraciones.

La implementación se realizó en un equipo con sistema operativo Microsoft Windows 10 Pro con procesador AMD A8-7410 APU y 6 GB RAM. Para evaluar el desempeño del algoritmo se hizo la implementación del modelo matemático del CVRP en el software de optimización Lingo. La Figura 6 presenta de manera general esta implementación, la cual utiliza el algoritmo de Branch \& Bound (B-and-B) para obtener una solución óptima.

\section{Resultados}

La Figura 7 presenta el esquema de ruteo obtenido para hacer la distribución a los 24 centros de reciclado diariamente. Este resultado se obtuvo en un tiempo de 30 segundos. Como se observa, se determinaron 4 rutas (4 vehículos) de distribución con 
el esquema de cobertura presentado en la Tabla 1. Estas rutas recorren una distancia Euclidiana de 1.3651 .

Tabla 1. Resultado de ruteo diario para distribuir a los 24 centros de reciclado.

\begin{tabular}{llrrrrrrr}
\hline Ruta 1 & 1 & 6 & 5 & 15 & 12 & 21 & 18 & 1 \\
Ruta 2 & 1 & 25 & 10 & 9 & 17 & 23 & 2 & 1 \\
Ruta 3 & 1 & 3 & 20 & 13 & 16 & 22 & 19 & 1 \\
Ruta 4 & 1 & 14 & 7 & 24 & 8 & 11 & 4 & 1 \\
\hline
\end{tabular}

En comparación, Lingo proporcionó una solución con 5 rutas y una distancia Euclidiana de 1.3347 en un tiempo aproximado de 30 minutos. El error de la solución obtenida mediante el meta-heurístico fue de [(1.3652 - 1.3347) /1.3347] $\times 100 \%=$ $2.28 \%$ lo cual es mínimo y representa una ventaja en cuanto a tiempo de obtención, principalmente para problemas de ruteo con mayor número de nodos. Finalmente, el meta-heurístico, al proporcionar una solución con menos rutas, puede favorecer la reducción en costos operativos.

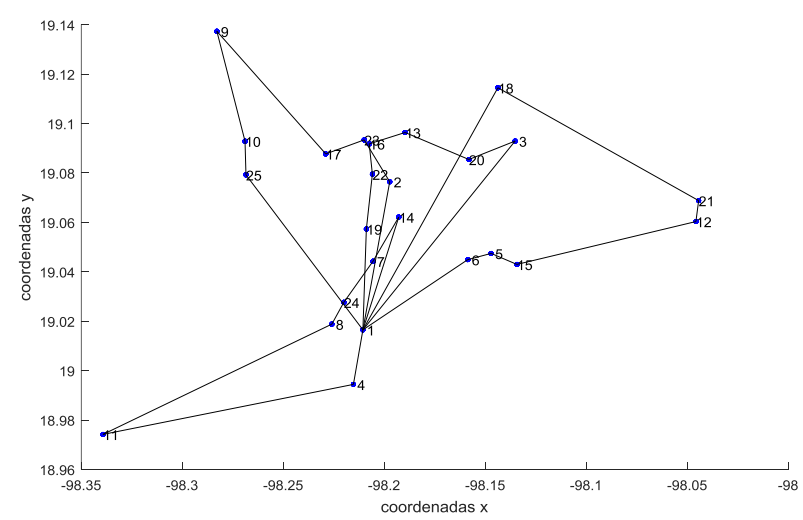

Fig. 7. Rutas determinadas para distribuir a los 24 centros de reciclado en Puebla.

\section{Conclusiones}

En el desarrollo del presente proyecto se conoció acerca del impacto de los residuos eléctricos y electrónicos en el medio ambiente y para el ser humano. De igual manera se conocieron las limitadas acciones e infraestructura para llevar a cabo el reciclado de estos desechos de manera efectiva.

Por lo anterior, se propuso una cadena de logística inversa para procurar que todos los desechos eléctricos y electrónicos que llegan a los servicios sanitarios de Puebla sean reciclados y de esta forma evitar lo más posible el contacto de las sustancias tóxicas que segregan con el medio ambiente.

Se determinó una solución para abarcar la zona metropolitana de Puebla, así como algunos puntos aleñados de manera efectiva. Con estas rutas se espera que se pueda transportar la tecno-basura hacia los centros de reciclado disponibles. 
Aunque este proyecto se enfoca exclusivamente a los desechos eléctricos y electrónicos, es recomendable desarrollar un proyecto similar enfocado hacia cada uno de los desechos de la basura.

Para ello, sería necesario que la población se organizara para separar todos y cada uno de los desechos que se generan, con la finalidad de que la separación y reciclado de la basura se garantice y sobre todo sea efectivo.

Como trabajo futuro se pretende estimar de manera más específica la cantidad de tecno-basura generada en la entidad y la disponibilidad de infraestructura para su transporte. De igual manera, incluir los diferentes desechos y calendarios de recolección y entrega para una mejor planeación en base a la densidad de transporte en la entidad.

\section{Referencias}

1. Aras, N., Aksen, D., Gönül Tanuğur, A.: Locating collection centers for incentive-dependent returns under a pick-up policy with capacitated vehicles. European Journal of Operational Research 191(3), 1223-1240 (2008)

2. Ayvaz, B., Bolat, B., Aydın, N.: Stochastic reverse logistics network design for waste of electrical and electronic equipment. Resources, Conservation and Recycling 104, pp. 391404 (2015)

3. Bazurto, J.A., Osorio, D., Usme, E., Satiba, S.: La tecno-basura un problema contemporáneo (16/11/2010), http://latecnobasura.blogspot.com/. Último acceso: 2018/12/19

4. Carroll, P., Keenan, P.: Chapter 10 - Decision Making Using Exact Optimization Methods in Sustainable Transportation. In: Faulin, J., Grasman, S.E., Juan, A.A., Hirsch, P. (eds.): Sustainable Transportation and Smart Logistics, pp. 263-283. Elsevier (2019)

5. Colprensa: Así afecta la basura electrónica a la salud y al medioambiente (25/12/2017). El Heraldo, https://www.elheraldo.co/salud/asi-afecta-la-basura-electronica-la-salud-y-almedioambiente-440278. Último acceso: 2018/12/18

6. David, S.: Sólo tres empresas reciclan electrónicos en Puebla (14/11/2017). Tierra Baldía: Comunicación y Periodismo, http://tierrabaldia.com.mx/noticia/1083/solo-tres-empresasreciclan-electronicos-en-puebla/. Último acceso: 2019/01/20

7. Dorantes, R.: Entregan 15 nuevos camiones recolectores de basura en V Carranza (15/05/2017). Excelsior, https://www.excelsior.com.mx/comunidad/2017/05/15/1163663. Último acceso: 2019/03/30

8. Secretaría General del Gobierno de los Estados Unidos Mexicanos: Ley General para la Prevención y Gestión Integral de los Residuos. Diario Oficial de la Federación (2018)

9. Duran, M., Caballero-Morales, S.O., Martínez-Flores, J.L., Cano-Olivos, P.: Improvement of Pick-Up Routes for an International Shipping Enterprise by Using a Heuristic Method. Revista Latino-americana de Inovação e Engenharia de Produção 4(6) 58-68 (2016)

10. González-Soto, E.: Tecno-basura agobia a Puebla y buscan mitigar sus efectos (1/04/2018) E-consulta.com, http://www.e-consulta.com/nota/2018-04-01/medio-ambiente/tecnobasuraagobia-puebla-y-buscan-mitigar-sus-efectos. Último acceso: 2019/03/30

11. Guarnieri, P., Camara-e-Silva, L., Levino, N.: Analysis of electronic waste reverse logistics decisions using Strategic Options Development Analysis methodology: A Brazilian case. Journal of Cleaner Production 133, pp. 1105-1117 (2016)

12. INEGI: México en Cifras (1/01/2018), http://www.beta.inegi.org.mx/app/areasgeograficas/? ag=21\#tabMCcollapse-Indicadores. Último acceso: 2018/04/30

13. Jabir, E., Panicker, V.V., Sridharan, R: Design and development of a hybrid ant colonyvariable neighborhood search algorithm for a multi-depot green vehicle routing problem. Transportation Research Part D: Transport and Environment 57, pp. $422-457$ (2017) 
14. Juan-Martín, M.: Ahora, Sony te da hasta $\$ 10,000$ por tu vieja televisión (1/04/2018). Paréntesis.com, https://www.parentesis.com/noticias/Informacion/Ahora_Sony_te_ da_hasta_10000_por_tu_vieja_television\#. Último acceso: 2019/03/30

15. Kannan, D., Diabat, A., Alrefaei, M., Govindan, K., Yong, G.: A carbon footprint based reverse logistics network design model. Resources, Conservation and Recycling 67, pp. 7579 (2012)

16. Mar-Ortiz, J., Adenso-Diaz, B., González-Velarde, J.L.: Design of a recovery network for WEEE collection: the case of Galicia, Spain. Journal of the Operational Research Society 62(8), 1471-1484 (2011)

17. Méndez, E.: México se inunda de tecno-basura; produce 300 mil toneladas al año (27/02/2011). Excélsior, https://www.excelsior.com.mx/node/717925. Último acceso: 2018/12/18

18. Prakash, C., Barua, M.K.: A combined MCDM approach for evaluation and selection of third-party reverse logistics partner for Indian electronics industry. Sustainable Production and Consumption 7, pp. 66-78 (2016)

19. Porter, N.: En México, sólo $10 \%$ de electrónicos se recicla de forma responsable (8/05/2018) La Capital, http://www.lacapital.com.mx/noticia/63156En_Mexico_solo_10_de_electronicos_se_recicla_de_forma_responsable. Último acceso: 2018/06/17

20. Redacción EC: ¿Qué países generan más basura electrónica? (20/04/2015). El Comercio. https://elcomercio.pe/tecnologia/actualidad/paises-generan-basura-electronica-374949. Último acceso: 2018/06/18

21. Ribas-Vila, I., Rubio, S.: Decisiones en el diseño de redes de logística inversa: propuesta de un modelo de decisión. En: Proceedings of the 7th International Conference on Industrial Engineering and Industrial Management - XVII Congreso de Ingeniería de Organización, pp. 1025-1033 (2013)

22. Shih, L.-H.: Reverse logistics system planning for recycling electrical appliances and computers in Taiwan. Resources, Conservation and Recycling 32(1), 55-72 (2001)

23. Sudarto, S., Takahashi, K., Morikawa, K., Nagasawa, K.: The impact of capacity planning on product lifecycle for performance on sustainability dimensions in Reverse Logistics Social Responsibility. Journal of Cleaner Production 133, pp. 28-42 (2016)

24. Valencia, M.: ¿Sabe qué pasa con los dispositivos electrónicos que dejó de usar? (21/04/2018). Dinero, http://www.dinero.com/internacional/articulo/las-consecuencias-dela-basura-electronica/257532. Último acceso: 2019/03/30

25. Yu, V.F., Redi, A.A.N.P., Hidayat, Y.A., Wibowo, O.J.: A simulated annealing heuristic for the hybrid vehicle routing problem. Applied Soft Computing 53, pp. 119-132 (2017) 